\title{
A selective medium for Pasteurella multocida and its use with animal and human specimens
}

\author{
DP KNIGHT, JANE E PAINE, DCE SPELLER \\ From the University Department of Clinical Microbiology, Bristol Royal Infirmary, Bristol BS2 $8 H W$
}

SUMMARY A selective medium (CGT medium), containing clindamycin, gentamicin, potassium tellurite and amphotericin B in 5\% horse-blood agar, allowed unimpaired growth of almost all strains of Pasteurella multocida, and $P$ pneumotropica, while inhibiting other bacteria that might be encountered in upper respiratory tract secretions. With its use, $P$ multocida was readily detected in oral swabs from four of $23 \mathrm{dogs}$, and 10 of 25 cats, but not detected in oral swabs from 47 human subjects. One of 500 sputum specimens yielded $P$ pneumotropica.

Pasteurella multocida is a common commensal of the upper respiratory tracts of domestic and other animals, and also causes disease in many species. ${ }^{1-4}$ It is encountered clinically in man mainly as a cause of acute inflammation in bites and scratches from dogs, cats and other animals, ${ }^{56}$ and it is also sometimes found as the predominant micro-organism in acute chest infections. ${ }^{7}$ Many of these cases have no known animal association ${ }^{8}$ and it has been suggested that there may be long-term carriage by humans. Isolation of $P$ multocida from human respiratory tract secretions may be difficult because of overgrowth by other bacteria, and because the prevalent Haemophilus strains may readily be confused with pasteurellae. In this investigation, we set out to devise and assess a selective medium which would be effective for such specimens.

\section{Material and methods}

Antibiotics and chemicals that showed differential activity against $P$ multocida strains and other bacteria commonly encountered in respiratory specimens were used alone and in combination in a base of heart infusion agar (Difco) with and without $5 \%$ defibrinated horse blood. The media were first tested by streaked inocula of the Pasteurella strains from the National Collection of Type Cultures, Central Public Health Laboratory, Colindale Avenue, London, which are listed in the Table, and with clinical isolates of $P$ multocida (identified by the methods of Cowan ${ }^{10}$ ) and clinical isolates of commensal bacteria. The medium finally chosen was

further tested by spot inoculation of $0.02 \mathrm{ml}$ of tenfold dilutions of overnight broth cultures of the bacteria, with, after overnight incubation at $37^{\circ} \mathrm{C}$ in air, observation of the highest dilution showing growth on blood agar and on the selective medium.

Oral specimens were taken with wool swabs (Medical Wire and Equipment Co) applied to the anterior surface of the gums and the interior of the cheek, from 48 animals ( 23 dogs and 25 cats) without evidence of infection, and from 47 volunteers, including children, members of an animal-loving charitable society, and kennel workers. Five hundred routine sputum specimens were homogenised with $\mathrm{N}$-acetyl cysteine (Sputasol, Oxoid). All the specimens were plated on the selective medium and on blood agar; the growths obtained after overnight incubation in air were compared and the growth on the selective medium, if any, investigated for the presence of $P$ multocida.

\section{Results}

The optimal composition finally established was: heart infusion agar with $5 \%$ defibrinated horse blood, containing clindamycin phosphate (Upjohn) $5.0 \mathrm{mg} / \mathrm{l}$, gentamicin sulphate (Roussel Laboratories) $0.75 \mathrm{mg} / \mathrm{l}$, potassium tellurite (BDH) $2.5 \mathrm{mg} / \mathrm{l}$, and amphotericin B (Sigma) $5.0 \mathrm{mg} / \mathrm{l}$. Quantitative comparison of the growth of selected cultures on this medium and on blood agar is shown in the Table. The selective medium permitted undiminished growth of 21/22 $P$ multocida clinical isolates and of NCTC 8489 ( $P$ multocida) and NCTC 8141 ( $P$ pneumotropica). One clinical isolate of $P$ multocida and NCTC 3195 ( $P$ multocida) grew poorly, as did NCTC 10365 ( $P$ haemolytica) and 
Growth of selected cultures on selective medium and blood agar: highest dilution of culture at which growth detectable, overnight incubation, $37^{\circ} \mathrm{C}$.

\begin{tabular}{|c|c|c|}
\hline \multirow[t]{2}{*}{ Bacterial strain } & \multicolumn{2}{|c|}{ Highest tenfold dilution of overnight broth culture at which growth detectable } \\
\hline & Blood agar & Selective medium \\
\hline $\begin{array}{l}\text { NCTC } 8489 \text { Pasteurella multocida } \\
\text { NCTC } 3195 P \text { multocida } \\
\text { NCTC } 10365 P \text { haemolytica } \\
\text { NCTC } 10219 P \text { haemolytica } \text { var ureae }\end{array}$ & $\begin{array}{l}-9 \\
-7 \\
-6 \\
-9\end{array}$ & $\begin{array}{l}-9 \\
-4 \\
-3 \\
-1\end{array}$ \\
\hline $\begin{array}{l}\text { Clinical isolates: } \\
P \text { multocida } 1 \\
P \text { multocida } 2 \\
P \text { multocida } 3 \\
P \text { multocida } 4 \\
\text { Pseudomonas aeruginosa } \\
\text { Proteus mirabilis }\end{array}$ & $\begin{array}{l}-7 \\
-8 \\
-8 \\
-7 \\
-7 \\
-7\end{array}$ & $\begin{array}{l}-6 \\
-7 \\
-8 \\
-8 \\
-7 \\
-2\end{array}$ \\
\hline
\end{tabular}

Other commensal bacteria were completely inhibited by selective medium.

NCTC 10219 (P haemolytica var ureae). Strains of other bacteria were completely inhibited, with the exception of Ps aeruginosa and Proteus spp. Only one of seven Proteus isolates grew well on the medium; the others were prevented from swarming and grew poorly. The medium was more effective than that of Morris ${ }^{11}$ in the suppression of haemophili. The medium retained its selective properties during storage at $4^{\circ} \mathrm{C}$ for four weeks, despite lysis of the blood component.

In animal specimens the presence of $P$ multocida was easily recognised on half plates of the selective medium, whereas on blood agar it was usually obscured by other bacteria (Fig. 1a and b). Of 23 dogs, $4(17 \%)$ yielded $P$ multocida from gum swabs; as did $10(40 \%)$ of 25 cats. Four cats were sampled in

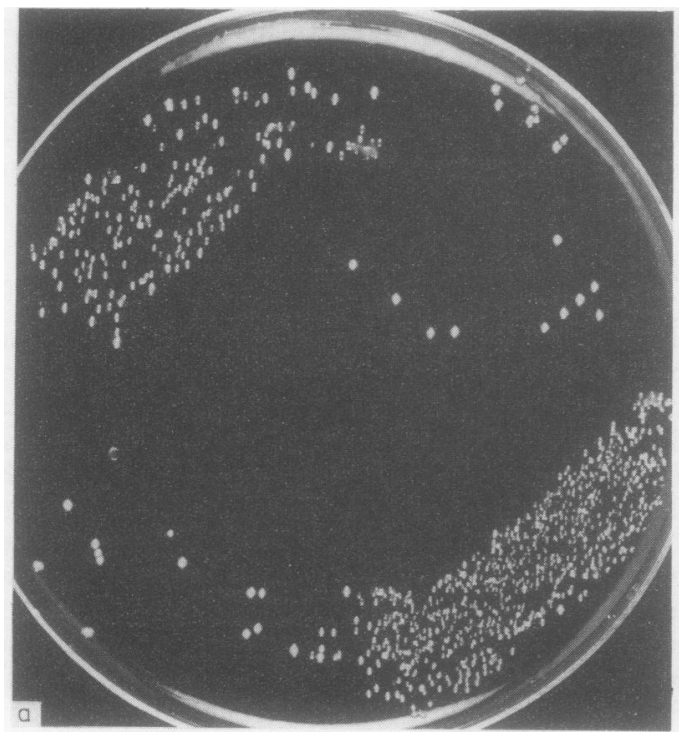

an identical manner at weekly intervals for five weeks, with very careful spreading of the inoculum and examination of the blood agar cultures for pasteurellae. $P$ multocida was detected on both selective medium and blood agar in three instances, on selective medium alone in 10 instances, and never on blood agar alone. None of the human oral specimens yielded $P$ multocida, but commensal mouth bacteria were effectively suppressed by the selective medium (Fig. 2a and b). Of 500 sputum specimens examined, only one yielded a pasteurella, $P$ pneumotropica. This specimen was obtained from a 42-year-old woman, owner of a pet dog, who had suffered an acute exacerbation of chronic bronchitis. $P$ pneumotropica was plentiful in this specimen and detected readily on non-selective media, as well as

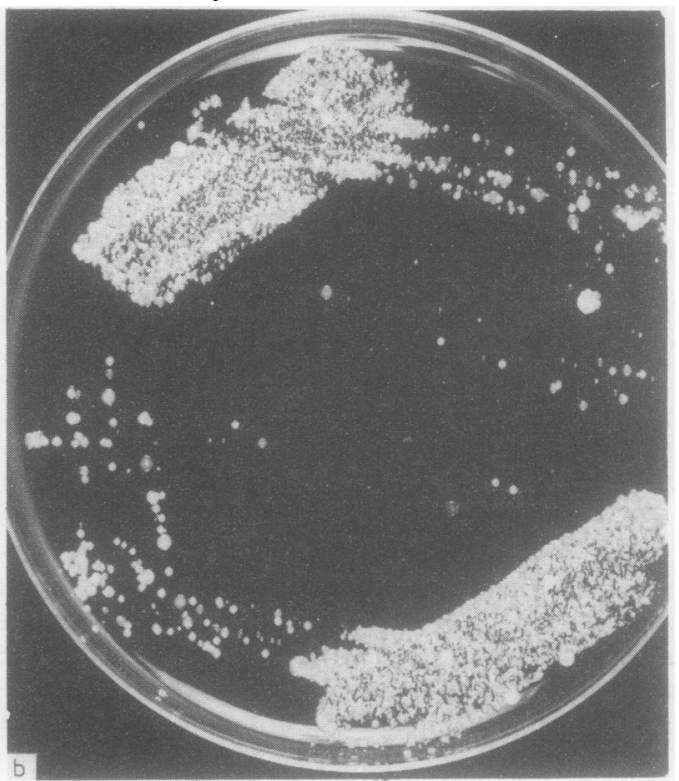

Fig. 1 (a) Oral swabs from two dogs plated on selective medium, showing a pure growth of Pasteurella multocida; (b) the same swabs plated on blood agar, showing a mixed growth of commensal bacteria. Both overnight incubation, $37^{\circ} \mathrm{C}$, in air. 

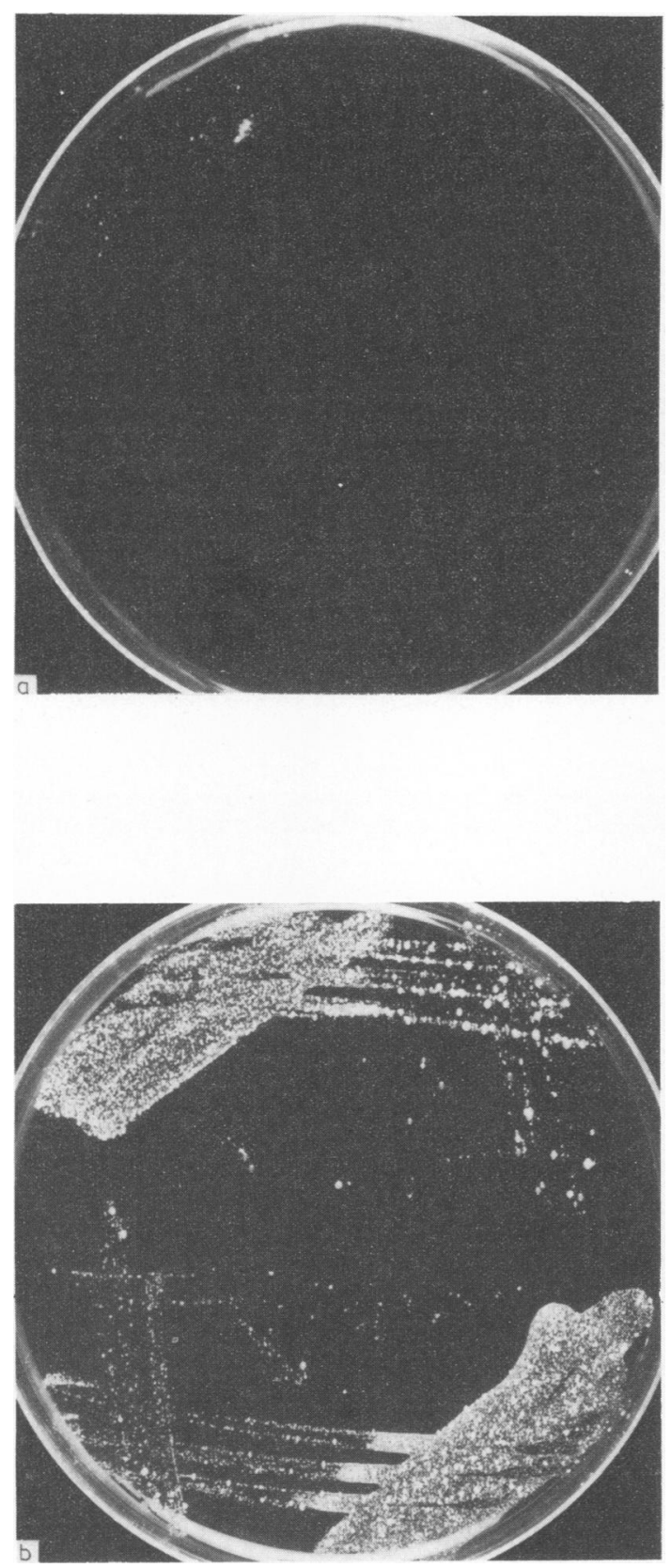

Fig. 2 (a) Oral swabs from two human subjects plated on selective medium, showing almost complete inhibition of commensal flora; (b) the same swabs plated on blood agar. Both overnight incubation, $37^{\circ} \mathrm{C}$, in air. selective medium. $P$ multocida in smaller amounts was consistently detectable on selective medium from sputum artificially inoculated with $P$ multocida and processed in a similar manner.

\section{Discussion}

In this investigation, the rate of isolation of $P$ multocida from animal specimens was of the same order as that obtained by others. Hawkins ${ }^{12}$ found $14 \%$ of gum and tonsil swabs from 50 dogs to be positive, and $52 \%$ of similar specimens from 50 cats. Woodgyer $^{1}$ found $42 \%$ of gum swabs from 199 cats to yield $P$ multocida. Smith ${ }^{13}$ obtained $P$ multocida from $54 \%$ of tonsil swabs from 111 dogs, and from $10 \%$ of nose swabs, but he was sampling dead animals, and the greater ease and efficiency of specimen taking may have increased the yield.

$P$ multocida has occasionally been isolated from the upper respiratory tract of healthy humans. Smith ${ }^{9}$ obtained it from the throats of $2 / 71$ veterinary students; in one it persisted for at least four months, and in the other $P$ multocida was detected by mouse inoculation only for a few days. Hubbert and $\operatorname{Rosen}^{8}$ reported five positive isolations from normal upper respiratory tracts. Henriksen and Jyssum $^{14}$ detected 10 Pasteurella strains in two years in nose and throat cultures from patients with respiratory complaints but in whom the pasteurellae could not be definitely adjudged the responsible pathogen. Six were $P$ multocida and four were $P$ haemolytica var ureae; the authors estimated the positivity rate in such specimens as 2-4 per thousand. Jones, ${ }^{15}$ in describing 15 isolations of $P$ haemolytica var ureae from $1 \%$ of sputum specimens received during eight months, reported only two isolations of $P$ multocida during this period. Our results in the short series of volunteers, including many who had close association with cats and dogs, again suggest that carriage of $P$ multocida is uncommon, unless there is an underlying pathological process.

Although the selectivity of the medium described here is not perfect, its practical usefulness in the culture of specimens from the upper respiratory tracts of animals has clearly been demonstrated, and it should prove valuable in extending the observations on human carriage.

We are indebted to the pet owners and volunteers, including members of the Patients Animal Welfare Society, for providing specimens; to Dr GR Smith and Dr TE Curtis for information and advice; and to Dr LR Hill of the National Collection of Type Cultures for confirming the identification of an atypical $P$ multocida isolate. 


\section{References}

' Woodgyer AJ. A survey of Pasteurella multocida in the oral cavities of cats. NZ J Med Lab Technol 1976;30:6-10.

${ }^{2}$ Furie RA, Cohen RP, Hartman BJ, Roberts RB. Pasteurella multocida infection. Report in urban setting and review of spectrum of human disease. NY State J Med 1980;80:1597-1602.

${ }^{3}$ Curtis PE, Ollerhead GE, Ellis CE. Pasteurella multocida infection of poultry farm rats. Vet Rec 1980;107:326-7.

${ }^{4}$ Curtis PE, Ollerhead GE. Investigation to determine whether healthy chickens and turkeys are oral carriers of Pasteurella multocida. Vet Rec 1981;108:206-7.

5 Tindall JP, Harrison CM. Pasteurella multocida infections following animal injuries, especially cat bites. Arch Dermatol 1972;105:412-6.

- Francis DP, Holmes MA, Brandon G. Infections after domestic animal bites and scratches. JAMA 1975;233:42-5.

${ }^{7}$ Olsen AM, Needham GM. Pasteurella multocida in suppurative diseases of the respiratory tract. Am J Med Sci 1952;224:7781.

${ }^{8}$ Hubbert WT, Rosen MN. Pasteurella multocida infection in man unrelated to animal bite. Am J Public Health 1970;60:110917.
- Smith JE. Studies on Pasteurella septica III. Strains from human beings. J Comp Pathol 1959;69:231-5.

${ }^{10}$ Cowan ST. Cowan \& Steel's manual for the identification of medical bacteria. 2nd ed. Cambridge University Press, 1974.

"Morris EJ. Selective media for some Pasteurella species. J Gen Microbiol 1958;19:305-11.

12 Hawkins LG. Local Pasteurella multocida infections. J Bone Joint Surg 1969;51A:363-6.

${ }^{13}$ Smith JE. Studies on Pasteurella septica I. The occurrence in the nose and tonsils of dogs. J Comp Pathol 1955;65:239-45.

${ }^{14}$ Henriksen SD, Jyssum K. A study of some pasteurella strains from the human respiratory tract. Acta Pathol Microbiol Scand 1961;51B:354-68.

is Jones DM. A pasteurella-like organism from the human respiratory tract. J Pathol Bacteriol 1962;83:143-51.

Requests for reprints to: Professor DCE Speller, Department of Microbiology, Bristol Royal Infirmary, Marlborough Street, Bristol BS2 8HW, England. 